\title{
TPC Together with Overlapped Time Domain Multiplexing System Based on Turbo Structure
}

\author{
${ }^{1}$ Hao Zheng, ${ }^{1}$ Mingjun Xing, ${ }^{2}$ Yutao Yue, ${ }^{3}$ Xue Li, ${ }^{1,4}$ Daoben Li, ${ }^{1}$ Chunlin Ji \\ ${ }^{1}$ Kuang-Chi Institute of Advanced Technology, Shenzhen China; \\ ${ }^{2}$ Shenzhen Kuang-Chi Innovative Technology Co. Ltd., Shenzhen China; \\ ${ }^{3}$ Shenzhen Kuang-Chi Advanced Technology Co. Ltd., Shenzhen China; \\ ${ }^{4}$ Beijing University of Posts and Telecommunications, Beijing, China;
}

hao.zheng@kuang-chi.org; mingjun.xing@kuang-chi.org; yutao.yue@kuang-chi.org; xue.li@kuang-

chi.org; daoben.li@kuang-chi.org; chunlin.ji@kuang-chi.org

\begin{abstract}
Overlapped time domain multiplexing (OVTDM) is a novel technique for utilizing inter-symbol interference (ISI) to benefit a communication system. We implement the OVTDM technique based on turbo structure and associate a turbo product code (TPC) to construct a novel coded turbo-structure OvTDM system. Two schemes of the iterative receiver and soft-input and soft-output (SISO) decoding algorithms are presented. Simulation results show an attractive advantage and performance of designed structures.
\end{abstract}

Keywords: OvTDM, turbo structure, TPC, SISO.

\section{Introduction}

It is well known that most traditional communication systems are designed based on Nyquist criterion [1], in which intersymbol interference (ISI) should be avoided between consequent symbols. In fact, the communication system without ISI is physically unrealizable. On the other hand, people tend to design a communication system with controlled ISI, such as Faster-than-Nyquist (FTN) signaling [2] and partial response signaling (PRS) [3]. However, these methods also treat the overlap between symbols as interference and do not really utilize it to collect the extra gain.

Based on ISI to benefit a communication system, overlapped time domain multiplexing (OvTDM) is proposed in [4]-[7]. The idea of OvTDM is to shift a data-weighted and band-limited multiplexing waveform in the time domain to achieve an overlap between different transmitted symbols and a high transmission rate. It can help to form a convolution structure among consequent symbols, so OvTDM can also be regarded as one kind of waveform convolution coding. Notice that, the overlapping process of OvTDM does not change the bandwidth of the basic multiplexing waveform [4]-[7]. So, the symbol bandwidth of OVTDM is the same with that of no overlapping. In the OvTDM system, these overlapped parts are never regarded as ISI but rather as a beneficial encoding constraint relationship that can provide corresponding gain. On the other hand, [7] explained that the essence of overlapped multiplexing systems is to construct independent parallel channels artificially. Therefore, compared with traditional communication systems, OvTDM can provide a greater system performance and higher capacity [4]-[9]. 
Hao Zheng, Mingjun Xing, Yutao Yue, Xue Li, Daoben Li, Chunlin Ji; Whisper: TPC Together with Overlapped Time Domain Multiplexing System Based on Turbo Structure, Transactions on Networks and Communications.

Volume 5 No. 6, December (2017); pp: 17-24

Most previous studies have focused on the single structure of the OvTDM system. However, [4][7] show that there is also a gap of capacity between the theoretical bound and the single structure OvTDM. One way to narrow the gap is employ the near-capacity forward-error-correction (FEC) codes. In addition, an alternative way is to improve the OVTDM system with extended the coding structure that inspired by some structured FEC codes [8]-[10], such as turbo codes. In order to further enhance the system performance, we combine two methods to construct a turbo-structure OvTDM with FEC codes. So, a coding system with three layers is formed, which contains the FEC code, the turbo structure and OvTDM respectively. In this paper, turbo product code (TPC) is employed as the FEC module. In comparison to another popular FEC code, the low-density parity-check code (LDPC) [11][12], TPC is suitable to be constructed with a shorter code length and requires fewer iterations for decoding [13][14], so it is more flexible in our structures.

This paper is organized as follows. The brief descirption of OvTDM and its turbo structure with FEC codes is given in section II. Section III discusses appropriate decoding algorithms. The comparative simulation study is shown in section IV. Finally, conclusions are made in section V.

\section{System Description}

\subsection{OvTDM Scheme}

In the OvTDM system, we artificially introduce ISI to form an overlap among different symbols. The mapping relationship between original bits and constellation symbols can follow the rule of ordinary modulation methods. Assuming the transmitted signals followed BPSK as $\mathbf{x}=\left[x_{0}, x_{1}, \cdots, x_{L-1}\right]$ with length $L$ and the multiplexing waveform as $h(t), t \in\left[0, T_{s}\right)$ with symbol duration $T_{s}$, then the transmitted signal after overlapping can be expressed as

$$
s(t)=\sum_{i=0}^{L-1} x_{i} h\left(t-i T_{s} / K\right)=\sum_{i=0}^{L-1} x_{i} h(t-i \Delta T)
$$

where $\Delta T=T_{s} / K$ is the time shift between symbols. In (1), $K$ is the number of overlapped symbols during $\Delta T$, which is named the overlapping coefficient or the constraint length. Notice that, the larger the coefficient $K$ is, the more serious the ISI introduced.

As discussed before, the OvTDM system is a waveform convolution coding system. So, maximum likelihood sequence detection (MLSD) [15] and maximum a posteriori (MAP) detection can be utilized to detect OvTDM signals. From the point of view of waveform convolution coding, the detection process can also be called OvTDM decoding.

Essentially, assuming the transmission channel is an additive white Gaussian noise (AWGN) channel, the OvTDM with the coefficient $K$ can be regarded as a system with $K$ independent parallel Gaussian channels. So, its capacity can totally achieve the sum of $K$ independent AWGN sub-channels [7].

\subsection{Turbo-Structure OvTDM with FEC}

Figure 1 shows the transmitter of the turbo-structure OvTDM with FEC. The coded sequence that has passed the FEC encoder and one interleaver is sent to the first OVTDM encoder for the $I$ channel. Meanwhile, the same sequence is sent to pass the other interleaver to form another sequence with a 
different order, which is encoded by the second OvTDM encoder for the $Q$ channel. The output sequences from both two OvTDM encoders are combined to form a complex sequence.

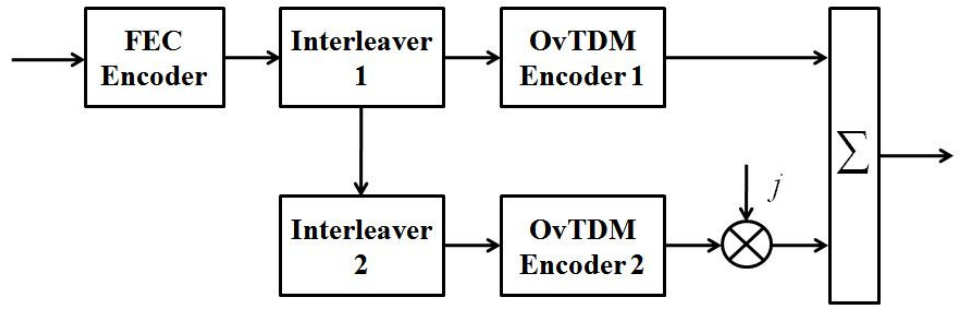

Figure 1. The transmitter structure of the turbo-structure OvTDM together with TPC.

The decoding process at the receiver is the key to the system design. It is based on the idea of iteration and the extrinsic information transformation [10]. During each iteration, the extrinsic information is exchanged between different decoders. The interleaver and the de-interleaver are employed to match the order of received sequences. Exchanging extrinsic information with low correlation can help to improve performance with the increase of iterations. Together with FEC, two schemes are addressed as follows:

Scheme A: After one round of decoding between two OvTDM decoders, the soft information is sent to the FEC decoder. Then the FEC decoder sends the soft information back to the OvTDM decoder. The model is shown in Figure 2. In this scheme, the FEC decoder needs to be involved in every iteration of the turbo structure.

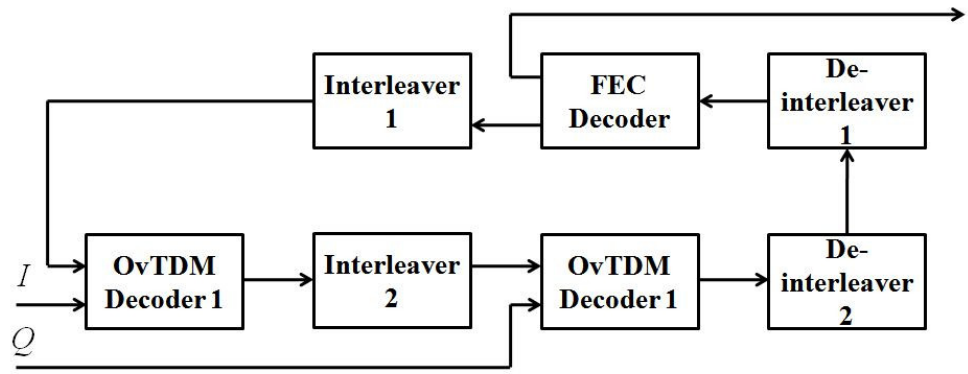

Figure 2. The receiver structure of the turbo-structure OvTDM together with TPC (Scheme A).

Scheme B: As shown in Figure 3, OvTDM decoders work iteratively and do not exchange soft information with the FEC decoder at first. After several iterations, the soft information is sent to the FEC decoder. Unlike in Scheme A, the soft information is only exchanged once between OvTDM decoders and the FEC decoder. 


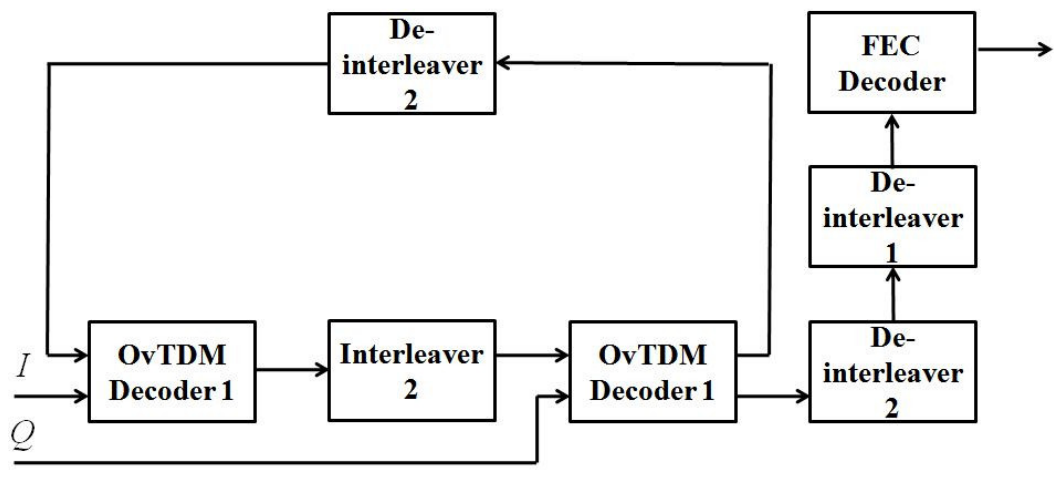

Figure 3. The receiver structure of the turbo-structure OvTDM together with TPC (Scheme B).

The Viterbi algorithm [16] is a good choice for detecting the OVTDM signals and selecting the possible sequence that is nearest to the received sequence [4][5]. However, in the turbo structure, we need to exchange the extrinsic information for original bits, so soft output detecting algorithms [17][18] are more appropriate.

\section{Decoding Algorithm}

\subsection{MAP Algorithm for OvTDM}

As discussed in the above sections, OvTDM utilizes the ISI as the encoding constraint. Thus, it can also be represented as a trellis graph [5]. The BCJR algorithm [17] is regarded as an optimal MAP method based on the trellis graph, so it can be modified to calculate the maximum a posteriori probability (APP) for OvTDM encoded bits.

Denoting the input bit at time $t$ as $x_{t}$ and the received sequence at the receiver as $\mathbf{r}$ with length $N$, the log-likelihood-ratio (LLR) of APP of $x_{t}$ is

$$
\lambda_{t}=\log \frac{\mathrm{p}\left(x_{t}=+1 \mid \mathbf{r}\right)}{\mathrm{p}\left(x_{t}=-1 \mid \mathbf{r}\right)}=\log \frac{\sum_{\left(S_{t-1}, S_{t}\right), x_{t}=+1} \mathrm{p}\left(S_{t-1}, S_{t}, \mathbf{r}\right)}{\sum_{\left(S_{t-1}, S_{t}\right), x_{t}=-1} \mathrm{p}\left(S_{t-1}, S_{t}, \mathbf{r}\right)}
$$

where $S_{t}$ and $S_{t-1}$ are assumed as states of time $t$ and $t-1$ based on the trellis graph. According to properties of OvTDM, $\mathrm{p}\left(S_{t-1}, S_{t}, \mathbf{r}\right)$ can be further expressed by

$$
\mathrm{p}\left(S_{t-1}, S_{t}, \mathbf{r}\right)=\alpha_{t}\left(S_{t-1}\right) \gamma_{t}\left(S_{t-1}, S_{t}\right) \beta_{t}\left(S_{t}\right)
$$

where $\alpha_{t}\left(S_{t-1}\right)$ and $\beta_{t}\left(S_{t}\right)$ can be calculated by forward and backward recursion:

$$
\begin{gathered}
\alpha_{t}\left(S_{t-1}\right)=\sum_{S_{t-1}} \alpha_{t-1}\left(S_{t-2}\right) \gamma_{t}\left(S_{t-1}, S_{t}\right) \\
\beta_{t}\left(S_{t}\right)=\sum_{S_{t+1}} \beta_{t+1}\left(S_{t+1}\right) \gamma_{t+1}\left(S_{t}, S_{t+1}\right)
\end{gathered}
$$

Assuming the corresponding output bit at time $t$ after OvTDM encoding as $y_{t}$, then

$$
\gamma_{t}\left(S_{t-1}, S_{t}\right)=\mathrm{p}\left(x_{t}\right) \mathrm{p}\left(r_{t} \mid y_{t}\right)
$$


It is worth noting that there is no input bit in the tail part of OvTDM. So, $\beta_{L}\left(S_{L}\right)$ can be initialized directly. In the AWGN channel,

$$
\beta_{L}\left(S_{L}\right)=\frac{1}{(\sqrt{2 \pi} \sigma)^{K}} \exp \left(-\frac{\sum_{i=L}^{N}\left(r_{t}-y_{t}\right)^{2}}{2 \sigma^{2}}\right)
$$

where $\sigma^{2}$ is the variance of noise.

Let LLR of a prior probability and the extrinsic information be $\mu_{t}$ and $e_{t}$, in the iterative decoder, the extrinsic information can be obtained by

$$
e_{t}=\lambda_{t}-\mu_{t}
$$

which is the output of the OvTDM decoding module.

\subsection{FBBA for TPC}

One mainstream method of TPC decoding algorithm is augmented list decoding (ALD) [13][14]. The key idea of ALD is to form a list including the most likely codewords. Based on ALD, the Fang-Battail-BudaAlgorithm (FBBA) [14] is an efficient SISO algorithm for TPC decoding to achieve near-optimum performance. FBBA is concluded as follows:

Step 1: Sort the received symbols $\mathbf{d}$ in a decreasing order according to the LLR metric $\mathbf{I}$.

Step 2: Permute the check matrix $\mathbf{H}$ according to the permutation pattern from the Step 1. Then, it has to be adjusted by Gauss-Jordan elimination to obtain a systematic one $\mathbf{H}^{\boldsymbol{\pi}}$ that is used to re-code the component codeword to generate a new one $\mathbf{c}^{\boldsymbol{\pi ( 0 )}}$.

Step 3: A codebook list is obtained through the reversal of certain positions of $\mathbf{c}^{\boldsymbol{\pi}(\mathbf{0})}$ and sorted in an increasing order according to

$$
Z\left(\mathbf{l}, \mathbf{c}^{\pi(\mathbf{i})}\right)=-\sum_{j=0}^{n-1} \log \frac{\mathrm{p}\left(l_{j} \mid c_{j}^{\pi(i)}\right)}{\mathrm{p}\left(l_{j} \mid c_{j}^{\pi(0)}\right)}
$$

where $\mathbf{c}^{\boldsymbol{\pi}(\mathbf{i})}$ and $n$ are denoted as the $i$ th codeword in the codebook list and the component codeword length.

Step 4: The soft output can be calculated by the first codeword $\mathbf{c}^{\pi(0)}$ and the opposite to the first codeword in $j$ th position in the codebook list.

$$
\rho_{j}=\frac{1}{4}\left(\left\|\mathbf{I}-\mathbf{c}^{\pi(\mathbf{o p p})}\right\|^{2}-\left\|\mathbf{I}-\mathbf{c}^{\pi(0)}\right\|^{2}\right)
$$

Following the above process, soft outputs can be calculated. Generally, four iterations are sufficient for the BER performance to converge.

\section{Simulation Study}

In this section, we need to investigate the performance through some comparative simulation. We choose the Chebyshev waveform with attenuation level $80 \mathrm{~dB}$ as the multiplexing waveform for OvTDM. Previous 
Hao Zheng, Mingjun Xing, Yutao Yue, Xue Li, Daoben Li, Chunlin Ji; Whisper: TPC Together with Overlapped Time Domain Multiplexing System Based on Turbo Structure, Transactions on Networks and Communications.

Volume 5 No. 6, December (2017); pp: 17-24

studies have proven and shown that overlapped multiplexing systems would not change the power spectrum of the basic multiplexing waveform [4]-[7]. Extended BCH $(64,57)$ is employed as the component codeword to construct a squared TPC. Thus, the code rate is $R_{T P C}=(57 / 64)^{2}=0.7932$. The AWGN channel is utilized as the transmission channel in the simulation.

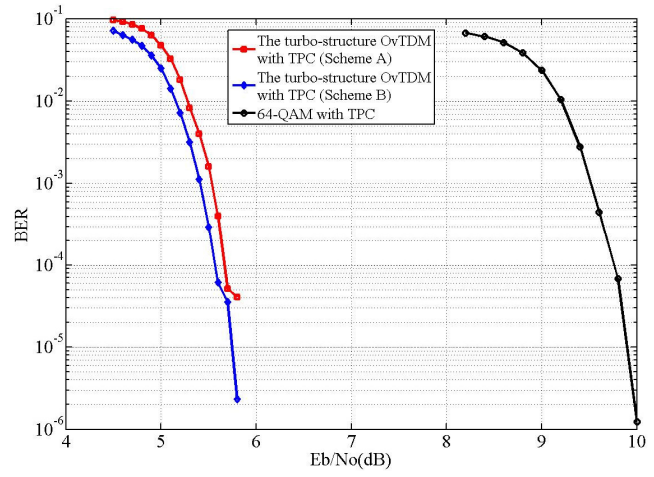

Figure 4. BER performance of the turbo-structure OvTDM $(K=6)$ and 64-QAM with TPC $(64,57)^{2}$.

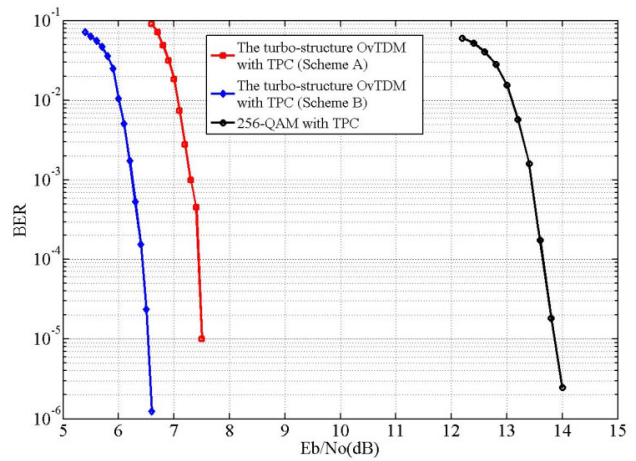

Figure 5. BER performance of the turbo-structure OvTDM $(K=8)$ and 256-QAM with $\operatorname{TPC}(64,57)^{2}$.

As mentioned before, the turbo-structure OvTDM uses the same information sequence for both I and $Q$ channels, so its equivalent coding rate with TPC is $R_{O v T}=1 / 2 \cdot R_{T P C} \cdot L / N$. When the length of the information sequence is large enough, $L / N \approx 1$, so we ignore it in the simulation. BPSK is used as the original modulation for the OvTDM system. Thus, the symbol efficiency of the turbo-structure OvTDM with TPC is $\eta_{O v T}=R_{O v T} \cdot 2 K=R_{T P C} K$ (bits/symbol). On the other hand, if we select $M$-ary QAM with TPC for comparison, its symbol efficiency is $\eta_{Q A M}=\log 2(M) R_{T P C}$ (bits/symbol). In order to do the comparative studies under the same symbol efficiency, we select $K=6$ and 64 -QAM in Figure 4 as well as $K=8$ and 256-QAM in Figure 5. The BER plots in both Figure 4 and Figure 5 show the significant advantage of the coded turbo-structure OvTDM. In Figure 4, the same symbol efficiency is 4.7592 (bits/symbol) and the required $E_{b} / N_{0}$ of 64 -QAM with TPC to achieve the BER $<10^{-5}$ is $10 \mathrm{~dB}$, but the turbo-structure OVTDM with TPC can achieve BER $<10^{-5}$ at $5.8 \mathrm{~dB}$. In Figure 5 , the same symbol efficiency is 6.3456 (bits/symbol) and the required $E_{b} / N_{0}$ of the turbo-structure with TPC using Scheme B to achieve the BER $<10^{-5}$ is $7.4 \mathrm{~dB}$ less than that of 256 -QAM with TPC.

Moreover, Figure 6 illustrates the comparison result of the capacity among different schemes when BER $<10^{-5}$. The capacity of the single structure OvTDM and the theoretical bound have been shown in [5] and [7]. In addition, we plot the corresponding capacity of the Shannon Bound [19]. In Figure 6, the turbostructure OVTDM with TPC shows an apparent improvement and advantage, compared with the single structure OvTDM and traditional communication systems. Also, it helps to narrow the discrepancy with the theoretical bound. 


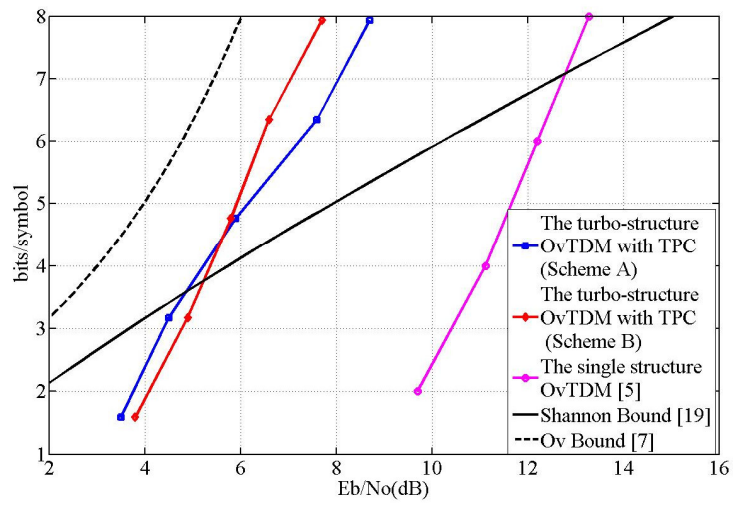

Figure 6. Comparison of capacity among different schemes.

\section{Conclusion}

This paper mainly focuses on structures and SISO decoding algorithms of the turbo-structure OvTDM with TPC, which demostrate a significant improvement over the single structure OvTDM. In addition, compared with the coded QAM system of the same symbol efficiency, the BER performance of the turbo-structure OvTDM with TPC is much better. Simulation results shows the advantage of the turbo-structure OvTDM with TPC in the communication scenario requiring high transmission rate at a relatively low $E_{b} / N_{0}$.

\section{ACKNOWLEDGMENTS}

The work was supported by State Key Laboratory of Meta-RF Electromagnetic Modulation Technology (2011DQ782011), Guangdong Key Laboratory of Meta-RF Microwave Radio Frequency (2011A060901010), Shenzhen Key Laboratory of Data Science and Modeling (CXB201109210103A), the introduction of innovative R\&D team program of Guangdong Province (NO. 2011D024) and Shenzhen Science and Technology Plan (JSGG20150917174734195, JSGG20150917174852555, JCYJ20151015165322766 and JCYJ20151015165557141).

\section{REFERENCES}

[1] Proakis, J. G., Digital Communications. 4th ed, Macgraw Hill, New York, 2001.

[2] Mazo, J. E., Faster-than-nyquist signaling. Bell Syst. Tech. J., 1975. 54(8): p. 1451-1462.

[3] Kabal, P. and Pasupathy, S., Partial-Response Signaling. Communications, IEEE Transaction on, 1975. 23(9): p. 921-934.

[4] Li, D., A novel high spectral efficiency waveform coding-OVTDM. International Journal of Wireless Communications and Mobile Computing, Special Issue: 5G Wireless Communication Systems, 2014. 2(41): p. 11-26.

[5] Li, D., Waveform Coding Theory of High Spectral Efficiency-OVTDM and Its Application. Science Press, Beijing, 2013. 
Hao Zheng, Mingjun Xing, Yutao Yue, Xue Li, Daoben Li, Chunlin Ji; Whisper: TPC Together with Overlapped Time Domain Multiplexing System Based on Turbo Structure, Transactions on Networks and Communications.

Volume 5 No. 6, December (2017); pp: 17-24

[6] Ji, C. and Liu, R., Study on a High Spectrum Modulation by Introducing Intersymbol Interference. in Signal Processing and Communications and Computing, 2016. Proceedings. 2016 IEEE International Conference on.

[7] Li, D., Channel Capacity on Additive White Gaussian Noise Channel under Overlapped Multiplexing Principle. Journal of Beijing University of Posts and Telecommunications, 2016. 39(6): p. 1-10.

[8] Dong, X., Research on the performance of OvTDM and Turbo-OvTDM technology application in multicarrier system. M.Sc. Dissertation, Beijing University of Posts and Telecommunication, 2013.

[9] Liu, B., Applications of overlapped multiplexing principle in telecommunications. Ph.D. Dissertation, Beijing University of Posts and Telecommunication, 2014.

[10] Berrou, C. and Glavieux, A., Near optimum error correcting coding and decoding: Turbo codes. Communications, IEEE Transaction on, 1996. 44(10): p. 1261-1271.

[11] Gallager, R., Low-density parity-check codes. Information Theory, IRE Transaction on, 1968. 8(1): p. 2128.

[12] Mackay, D. J. C. and Neal, R. M., Near shannon limit performance of low density parity check codes. Electronic Letters, 1977. 33(6), p. 457-458.

[13] Pyndiah, R., Near optimum decoding of product codes: block turbo codes. Communications, IEEE Transaction on, 1998. 46(8): p. 1003-1010.

[14] Fang, J., Buda, F. and Lemois, E., Turbo Product Code: a well suitable solution to wireless packet transmission for very low error rates. in Turbo Codes \& Related Topics, 2000. Proceedings. 2nd International Symposium on.

[15] Forney, G. D., Maximum Likelihood Sequence Estimation of Digital Sequences in the presence of intersymbol interference. Information Theory, IEEE Transaction on, 1972. 18(3): p. 363-378.

[16] Viterbi, A. J., Error bounds for convolutional codes and an asymtotically optimum decoding algorithm. Information Theory, IEEE Transaction on, 1967. 13(2): p. 260-269.

[17] Bahl, L. R., Cocke, J., Jelinek, F. and Raviv, J., Optimal decoding of linear codes for minimizing symbol error rate. Information Theory, IEEE Transaction on, 1974. 20(2): p. 284-287.

[18] Ji, C., On Sequential Learning for Parameter Estimation in Particle Algorithms for State-Space Models. International Journal of Statistics and Probability, 2017. 6(1): p. 13-23.

[19] Shannon, C. E., A mathematical theory of communication. Bell System Technical Journal, 1948. 27(7): p. 379-423. 